\title{
LONG-TERM SURVIVAL OF PATIENTS WITH CORONARY ARTERY DISEASE AND LEFT VENTRICULAR DYSFUNCTION: IMPLICATIONS FOR THE ROLE OF MYOCARDIAL VIABILITY ASSESSMENT IN MANAGEMENT DECISIONS
}

\author{
Marcelo F. Di Carli, MD \\ Jamshid Maddahi, MD \\ Sepehr Rokhsar, BA ${ }^{\mathrm{a}}$ \\ Heinrich R. Schelbert, MD \\ Daniela Bianco-Batlles, $\mathrm{MD}^{\mathrm{b}}$ \\ Richard C. Brunken, MD* \\ Barbara Fromm, MA $^{\mathrm{b}}$
}

Objectives: Our purpose was to evaluate the long-term benefit of myocardial viability assessment for stratifying risk and selecting patients with low ejection fraction for coronary artery bypass grafting and to determine the relation between the severity of anginal symptoms, the amount of ischemic myocardium, and clinical outcome. Methods: We studied 93 consecutive patients with severe coronary artery disease and low ejection fraction (median, 25\%) who underwent positron emission tomography to delineate the extent of perfusion-metabolism mismatch (reflecting hibernating myocardium) for potential myocardial revascularization. Median followup was 4 years (range, 0 to 6.2 years). Results: Fifty patients received medical therapy, and 43 patients underwent bypass grafting. In Cox survival models, heart failure class, prior myocardial infarction, and positron emission tomographic mismatch were the best predictors of survival. Patients with positron emission tomographic mismatch receiving bypass grafting had improved 4-year survival compared with those on medical therapy $(75 \%$ versus $30 \% ; P=.007)$ and a significant improvement in angina and heart failure symptoms. In patients without positron emission tomographic mismatch, bypass grafting tended to improve survival and symptoms only in those patients with severe angina $(100 \%$ versus $60 \% ; P$ $=.085$ ), whereas no survival advantage was apparent in patients with minimal or no anginal symptoms $(63 \%$ versus $52 \% ; P=.462)$. Conclusions: Patients with low ejection fraction and evidence of viable myocardium by positron emission tomography have improved survival and symptoms with coronary bypass grafting compared with medical therapy. In patients without evidence of viability, survival and symptom improvement with bypass grafting are apparent only among those patients with severe angina. (J Thorac Cardiovasc Surg 1998; 116:997-1004)
$D$ atients with coronary artery disease and severe left ventricular dysfunction have a poor prognosis when treated with medical therapy alone. ${ }^{1}$ Surgical revascu-

From the Division of Nuclear Medicine, Department of Medical and Molecular Pharmacology, UCLA School of Medicine, Los Angeles, Califa; and the Departments of Internal Medicine and Radiology, Wayne State University School of Medicine, Detroit, Mich. ${ }^{b}$

Dr Di Carli is the recipient of a Scientist Development Grant from the American Heart Association, Dallas, Texas.

Received for publication Feb 3, 1998; revisions requested April 29, 1998; revisions received June 4, 1998; accepted for publication July 21, 1998.

Address for reprints: Marcelo F. Di Carli, MD, Division of Cardiology, Harper Hospital, 3990 John R., Detroit, MI 48201.

*Current address: Department of Nuclear Medicine, Cleveland Clinic Foundation, 9500 Euclid Ave, Cleveland, OH 44195.

Copyright (C) 1998 by Mosby, Inc.

0022-5223/98 \$5.00+ $0 \quad \mathbf{1 2 / 1 / 9 3 2 9 4}$ larization appears to afford a long-term survival benefit in selected patients with low ejection fraction. ${ }^{2-4}$ However, the selection of patients with low ejection fraction for revascularization remains controversial because of the high surgical risk. ${ }^{5}$

In some patients with impaired left ventricular function, myocardial dysfunction results from infarction with attendant necrosis and scar formation. However, in many patients such myocardial dysfunction may be reversible with revascularization, ${ }^{6-11}$ otherwise referred to as hibernating and/or stunned myocardium. ${ }^{12-14}$ This has important implications for patients with low ejection fraction, in whom severe heart failure may be attributed to severe, widespread hibernation (or stunning or both) rather than to necrosis of a critical mass of myocardium. ${ }^{15}$ Previous studies have shown that identification of potentially reversible myocardial dysfunction in these patients 
Table I. Baseline characteristics of study patients

\begin{tabular}{|c|c|c|c|}
\hline Variable & $\begin{array}{l}\text { Medical } \\
\text { therapy } \\
(n=50)\end{array}$ & $\begin{array}{c}\text { Revascularization } \\
\quad(n=43)\end{array}$ & P value \\
\hline $\operatorname{Age}(\mathrm{yr})^{*}$ & $69(60.3,73.6)$ & $68(61.2,73.5)$ & .78 \\
\hline Male sex (No.; \%) & $40(80)$ & $38(88)$ & .27 \\
\hline $\begin{array}{l}\text { Angina class III-IV }{ }^{\dagger} \\
\text { (No.; \%) }\end{array}$ & $15(30)$ & $19(44)$ & .16 \\
\hline Diabetes (No.; \%) & $5(10)$ & $7(16)$ & .37 \\
\hline Hypertension (No.; \%) & $6(12)$ & $11(26)$ & .06 \\
\hline Prior MI (No.; \%) & $32(64)$ & $31(72)$ & .41 \\
\hline $\begin{array}{l}\text { CHF class III-IV } \\
(\text { No.; \%) }\end{array}$ & $31(62)$ & $32(74)$ & .50 \\
\hline LV ejection fraction* & $0.25(0.2,0.3)$ & $0.25(0.2,0.3)$ & .61 \\
\hline One vessel ${ }^{\S}($ No.; \%) & $9(18)$ & $8(19)$ & .94 \\
\hline Two vessels (No.; \%) & $28(56)$ & $16(37)$ & .09 \\
\hline Three vessels (No.; \%) & $12(24)$ & $16(37)$ & .18 \\
\hline Left main (No.; \%) & $1(2)$ & $3(7)$ & .33 \\
\hline Extent of PET mismatch ${ }^{*}$ & $12(6.4,17.6)$ & $18(7.5,28.8)$ & .20 \\
\hline
\end{tabular}

$M I$, Myocardial infarction; $C H F$, congestive heart failure; $L V$, left ventricular.

"Data presented are median (lower, upper, quartiles).

${ }^{\dagger}$ Canadian Cardiovascular Society.

"New York Heart Association.

${ }^{\S}$ Reflect proximal left anterior descending coronary artery disease.

appears effective for identifying those who will benefit most from revascularization. ${ }^{16-21}$ Although these studies suggest that myocardial viability assessment may be beneficial for predicting short-term outcome, the longterm prognostic benefit of such an approach remains to be defined. Further, the relation between the severity of anginal symptoms, the amount of ischemic myocardium, and outcome has not been well established.

Our purpose was to evaluate the long-term benefit of myocardial viability assessment for stratifying risk and selecting patients with low ejection fraction for coronary artery bypass grafting (CABG) and to determine the relation between the severity of anginal symptoms, the amount of ischemic myocardium, and clinical outcome.

\section{Methods}

Patient population and data collection. All patients who were undergoing myocardial viability assessment from February 1989 through November 1991 for any symptom of coronary artery disease, who had moderate-to-severe left ventricular dysfunction (ejection fraction, <40\%), and who were potential candidates for myocardial revascularization were selected for this study. Patients were referred by University of California, Los Angeles faculty and by physicians from the Los Angeles area, the majority of whom were cardiologists or cardiothoracic surgeons.

All patients underwent coronary angiography by standard techniques. The criterion for evaluating the angiographic extent of coronary artery disease was either a $70 \%$ or more stenosis in the proximal left anterior descending, circumflex, or right coronary artery or their major branches, or $50 \%$ or more stenosis of the left main coronary artery. The left ventricular ejection fraction was calculated from biplane contrast left ventriculography in 70 patients and 2-dimensional echocardiography in 23 patients. The results are summarized in Table I.

Evaluation of myocardial viability. Myocardial viability was evaluated with positron-emission tomography (PET) with ${ }^{13} \mathrm{~N}$-ammonia $(20 \mathrm{mCi})$ as a tracer of blood flow and ${ }^{18} \mathrm{~F}$-deoxyglucose (FDG; $10 \mathrm{mCi}$ ) as an indicator of glucose use. Imaging was performed on a whole body PET tomograph (model 931-108; Siemens-Computer Technology, Inc, Knoxville, Tenn). Studies were acquired in the glucoseloaded state, after oral administration of $50 \mathrm{~g}$ of glucose.

The analysis of PET images was previously described in detail. ${ }^{16}$ Briefly, analysis of regional myocardial perfusion, glucose uptake, and their relationship was performed with a 20-segment model. Visual analysis was as follows: Corresponding ${ }^{13} \mathrm{~N}$-ammonia and FDG images were scored semiquantitatively in all 20 segments by consensus visual analysis of 2 observers who were blinded to the rest of the patient's data. Segments with a perfusion defect were classified as a match, when both ${ }^{13} \mathrm{~N}$-ammonia and FDG uptake were concordantly reduced, or a mismatch when segmental FDG uptake was increased relative to perfusion. The total myocardial extent of PET patterns was determined by dividing the number of segments that showed a match or mismatch pattern by 20 (ie, total number of left ventricular segments). Quantitative analysis was as follows: Regional ${ }^{13} \mathrm{~N}$-ammonia and FDG uptake were also evaluated quantitatively with volume-weighted polar maps, as described previously. ${ }^{16}$ Segments classified as a PET match on visual analysis had on average a concordant reduction in ${ }^{13} \mathrm{~N}$-ammonia and FDG uptake on quantitative analysis (mean $\pm \mathrm{SD}, 56 \% \pm 19 \%$ versus $57 \% \pm 18 \%$, respectively; $P=.712$ ). Conversely, myocardial segments classified as a PET mismatch by visual analysis had, on average, a significant relative increase (to perfusion) in FDG uptake on quantitative analysis (mean $\pm \mathrm{SD}, 57 \% \pm$ $15 \%$ versus $93 \% \pm 20 \%$, respectively; $P<.001$ ).

Follow-up. The decision to treat a patient with revascularization or medical therapy was made by the primary physicians and the patients on the basis of clinical grounds. Because of refractory symptoms, 5 patients who initially received medical therapy underwent late revascularization $(>6$ months after the PET study), and 2 patients received a heart transplant. These patients were censored from further analysis at the time of revascularization or heart transplantation. Patients were followed for a median of 3.8 years (range, 0 to 6.2 years). Information on the vital status of patients was obtained by telephone contact with the patient, relatives, and referring physicians and from review of hospital records and death certificates by an investigator who was blinded to the rest of the data. The cause of death was determined from death certificates, hospital records, or autopsy reports. Deaths caused by acute myocardial infarction, ventricular arrhythmia, and congestive heart failure were considered cardiac deaths. Death was considered sudden when it was unexpected 
and was witnessed to occur within 1 hour of the onset of symptoms.

Statistical analysis. Baseline differences in important prognostic factors between the medical and revascularization groups were assessed with $\chi^{2}$ test for discrete variables and the Wilcoxon rank sum test for continuous variables. Analysis of the primary end point is based on an intention-totreat strategy. Survival curves were constructed from standard life table calculations after adjustment for differences in prognostic factors with the Cox proportional hazards model. ${ }^{22}$ For construction of survival curves, patients were assigned to the PET mismatch group when more than 5\% of the left ventricle had a perfusion-metabolic mismatch pattern, which was found to have the best trade-off between sensitivity and specificity for predicting cardiac death by receiver operating-characteristic analysis. ${ }^{16}$ The Cox model was used to determine the influence of multiple factors on survival. Treatment method (ie, revascularization or medical therapy) was then added as a covariate in the Cox model so that survival differences between treatments could be tested. $P$ values less than .10 also are reported because they were taken to indicate trends toward statistical significance.

\section{Results}

Ninety-three patients met the inclusion criteria, of whom 50 patients received medical therapy and 43 patients underwent surgical revascularization. The baseline characteristics of the study group are summarized in Table I. There were some imbalances between the 2 groups, none of which reached statistical significance. The surgical group tended to have more male patients and more patients with severe angina, advanced heart failure, diabetes, hypertension, prior myocardial infarction, and larger areas of PET mismatch. Importantly, there was a significant overlap between the 2 groups with respect to important prognostic variables, including age, extent of coronary artery disease, and degree of left ventricular dysfunction.

Operative characteristics of patients undergoing CABG. All 43 patients underwent complete revascularization on the basis of the anatomic distribution of coronary stenoses and a review of operative reports. CABG was performed at many centers throughout Southern California. Eleven patients (26\%) had a history of previous CABG. Standard cardiopulmonary bypass and moderate hypothermia $\left(28^{\circ} \mathrm{C}\right)$ were used in all patients. Cold blood cardioplegia was used in 40 patients (93\%), and crystalloid cardioplegia was used in 3 patients (7\%). Cardioplegic solution was delivered via the antegrade route in 17 patients (40\%), whereas combined antegrade and retrograde (coronary sinus) delivery of cardioplegic solution was used in $26(60 \%)$ patients. The mean $( \pm \mathrm{SD})$ number of grafts for the group was $3.9( \pm 1.1)$. Arterial grafts were used in 10 patients $(23 \%)$. Thirteen patients $(30 \%)$ required intraaortic balloon pump support after the operation.

Survival analysis. Thirty-seven $(40 \%)$ deaths, all of which were cardiac in origin, occurred during the follow-up period. Revascularization $(P=.009)$ and the extent of PET mismatch $(P=.012)$ were the only statistically significant predictors of survival in univariate analysis. Sex, presence of Q waves on the resting electrocardiogram, diabetes, hypertension, extent of PET match, type of cardioplegia or its route of delivery, use of arterial conduits, and the extent of coronary artery disease on angiography were not significant predictors of survival in the univariate analysis.

A stepwise Cox model analysis was performed to determine the prognostic contribution of mismatch when covariates with borderline significance in the univariate analysis were included in the model. The variables considered in this analysis were age, angina, heart failure class, ejection fraction, prior myocardial infarction, extent of PET mismatch, type of treatment, and interaction between treatment and extent of PET mismatch, treatment and heart failure class, and treatment and prior myocardial infarction. In this model, only prior myocardial infarction (relative risk and $95 \%$ confidence interval; $1.49,1.02$ to $2.18 ; P=.038$ ), heart failure class $(1.59,0.94$ to $2.71 ; P=.086)$, and the extent of PET mismatch $(2.52,1.19$ to $5.32 ; P=.016)$ were significant predictors of outcome. These variables continued to be independent predictors of outcome when treatment method was entered into the Cox model so that differences between $\mathrm{CABG}$ and medical therapy groups could be tested. After adjusting for these important prognostic factors, survival was significantly better over time with revascularization than with medical therapy alone (Fig 1). The estimated adjusted survival probability for the 50 patients on medical therapy was $77 \%$, $46 \%$, and $33 \%$ after 1,3 , and 5 years of follow-up, respectively. In contrast, the estimated adjusted survival probability for the 43 patients undergoing revascularization was $95 \%, 84 \%$, and $78 \%$ after the same time intervals $(P=.0002)$. The improved survival with CABG was most apparent among patients with a left ventricular ejection fraction $20 \%$ or more (Fig 2).

Severity of anginal symptoms, amount of viable myocardium, and clinical outcome. To further evaluate the value of myocardial viability assessment for risk stratification of patients with left ventricular dysfunction, the study patients were subgrouped by presence or absence of mismatch (an important prognostic factor by multivariable analysis) on their preoperative PET images. 
Unadjusted

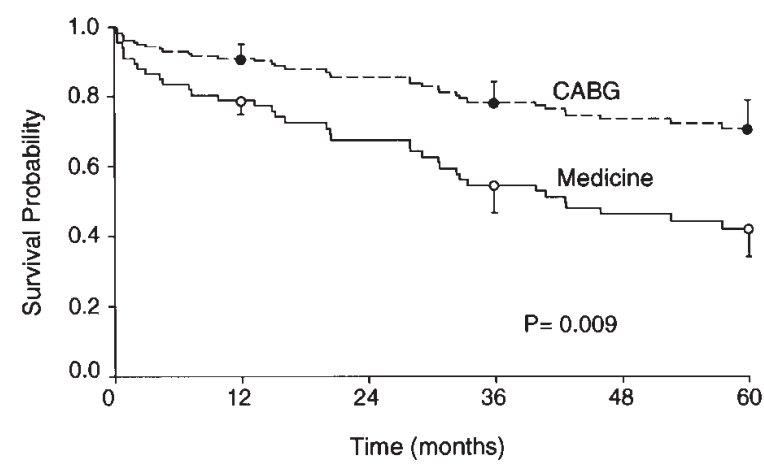

Adjusted

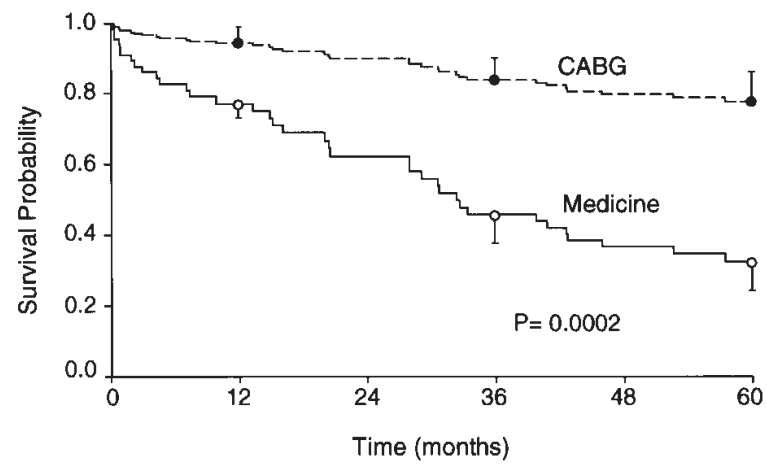

Patients at risk
$\begin{array}{ll}\text { CABG } & 43 \\ \text { Medicine } & 50\end{array}$

38

34
28

32

Fig 1. Plot shows Kaplan-Meier estimated survival probabilities for patients with left ventricular ejection fraction less than $40 \%$ treated medically and with CABG. The left panel shows the unadjusted (for baseline prognostic factors) survival estimates, and the right panel shows the adjusted survival estimates.

There were 25 deaths among patients receiving medical therapy, of which 11 deaths occurred in the group with PET mismatch. The mode of death was sudden in 8 of $11(73 \%)$ patients with PET mismatch and in 4 of $14(29 \%)$ patients without mismatch $(P=.031)$. The estimated 4-year adjusted survival probability of all medically treated patients was poor, but it was significantly lower among those with PET mismatch as compared with those without such a mismatch: $30 \%$ versus $52 \%$, respectively ( $P=.003$; Fig 3 ). Interestingly, most of the deaths in the group with PET mismatch occurred early in the course of the follow-up period.

There were 12 deaths among patients undergoing revascularization. Four of these deaths occurred during the 30-day perioperative period, resulting in a perioperative mortality of $9.3 \%$. The 4 in-hospital deaths in our study were due to low output state in 3 patients and sepsis with multi-organ failure in 1 patient. In these patients, ejection fraction ranged from $11 \%$ to $25 \%$; all had class III or IV heart failure, and 2 patients also had severe angina. Eleven of $43(26 \%)$ patients in the surgical group underwent repeat $\mathrm{CABG}$, of whom 2 patients died within the 30-day postoperative period. The 30-day perioperative mortality of patients undergoing repeat $\mathrm{CABG}$ was higher, although not statistically significant, than that of those receiving their first operation (2 of 11 [18.2\%] versus 2 of 32 [6.3\%], respectively; $P=.266$ ). The estimated 4 -year adjusted survival probability of revascularized patients with a preoperative PET mismatch was similar to those without a mismatch $(75 \%$ versus $77 \% ; P=.612)$.
Among patients with PET mismatch, those undergoing revascularization had a higher estimated 4-year adjusted survival probability as compared with those receiving medical therapy alone $(75 \%$ versus $30 \% ; P=$ .007 ; Fig 3). This survival benefit was apparent in patients with severe angina and in those with minimal or no anginal symptoms (Fig 4). Among patients without PET mismatch, revascularization tended to improve survival compared with medical therapy only among those patients with severe angina (4-year adjusted survival probability, $100 \%$ versus $60 \% ; P=.085)$, whereas no survival benefit was apparent in patients with minimal or no anginal symptoms (4-year adjusted survival probability, $63 \%$ versus $52 \% ; P=.462$; Fig 4 ).

Changes in anginal and heart failure symptoms. In the medically treated patients, there was no significant change in the number of angina-free patients during the follow-up period (30\% versus $24 \% ; P=.221$ ). In contrast, medical treatment provided some relief in the severity of heart failure symptoms during the course of the follow-up period (median [25th, 75th] heart failure class from baseline to follow-up, $3[2,3]$ to $2.5[2,3] ; P=.053)$. In the surgical patients, there was a significant increase in the number of angina-free patients during the follow-up period (44\% versus $7 \%$; $P<.001)$. Likewise, revascularization provided a significant relief in the severity of heart failure symptoms (median [25th, 75th] heart failure class from baseline to follow-up, 3 [2, 4] to $2[1,3]$; $P<.001)$. This was most apparent in patients with PET mismatch $(3[2.75,4]$ to $2[1,2] ; \mathrm{P}<.001)$. In patients without PET mismatch, 
revascularization tended to improve heart failure symptoms only when those were associated with severe angina, (3 [2, 3] to $2[2,3] ; P=.068)$, whereas no improvement was observed among patients in whom heart failure was associated with minimal or no angina (3 $[2.75,3.25]$ to $3[2,3] ; P=.108)$.

\section{Discussion}

Patients with coronary artery disease and severely impaired cardiac function have a poor prognosis when treated with medical therapy alone. ${ }^{1}$ In selected patients, CABG may improve survival and symptoms. $^{2-5,15}$ However, operative deaths are high, and much controversy remains about the proper selection of patients with severe left ventricular dysfunction for revascularization, especially among those patients with heart failure symptoms and minimal or no angina. ${ }^{5}$ The findings of this study suggest that in patients with evidence of viable myocardium, as assessed by the PET mismatch pattern, the 4-year adjusted survival with surgical revascularization is better compared with medical therapy alone (Fig 3). In patients without such evidence of viability, revascularization tended to improve survival over medical therapy only among those patients with severe angina, whereas no survival advantage was apparent in patients with minimal or no anginal symptoms (Fig 4). These results extend the observations of previous studies ${ }^{16-20}$ from our group and others in 2 important ways: by demonstrating the longterm benefit of the use of myocardial viability information for stratifying risk and selecting patients with impaired cardiac function for revascularization and by describing the interrelation between the severity of anginal symptoms, the presence of viable myocardium, and clinical outcome.

In this study, the overall adjusted survival of patients who received medical therapy alone was very poor, consistent with previous studies of the natural history of coronary artery disease. ${ }^{1-4}$ However, long-term survival was poorer among those patients with viable myocardium, as assessed by the PET mismatch pattern (Fig 3). This finding confirms and extends the results of previous reports, demonstrating that 1-year event-free survival in patients receiving medical therapy appears to be better among those patients without evidence of dysfunctional but viable myocardium, as determined by PET, thallium, or echocardiographic criteria. ${ }^{16-20}$ Although the reason for the association between viable myocardium and adverse outcome in medically treated patients is not well understood, it could be explained by the higher risk of ischemic events (ie, myocardial ischemia, infarction, and ventricular arrhythmia) when a severely stenosed coronary artery supplies an area of
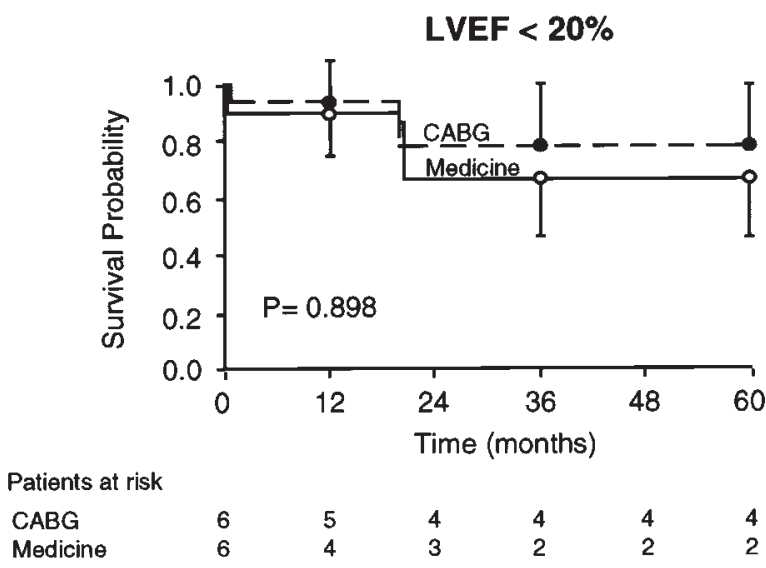

$\begin{array}{lllllll}\text { CABG } & 6 & 5 & 4 & 4 & 4 & 4 \\ \text { Medicine } & 6 & 4 & 3 & 2 & 2 & 2\end{array}$
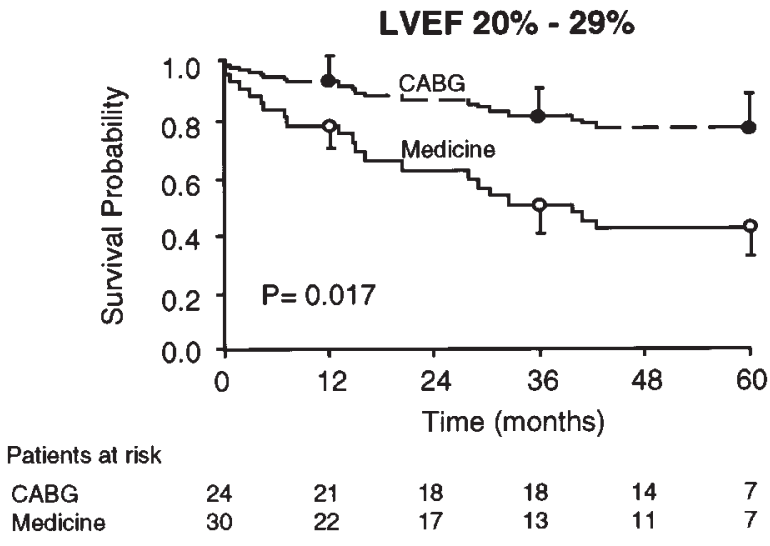

LVEF $\mathbf{3 0} \%-39 \%$

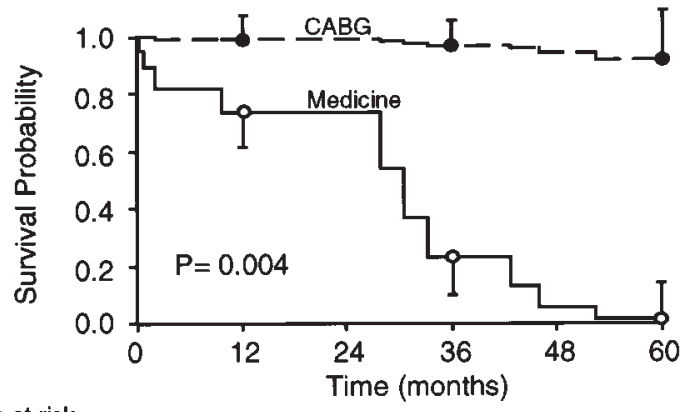

Patients at risk

$\begin{array}{lrrrrrr}\text { CABG } & 13 & 12 & 12 & 12 & 9 & 4 \\ \text { Medicine } & 14 & 9 & 8 & 5 & 2 & 1\end{array}$

Fig 2. Plot shows adjusted Kaplan-Meier estimated survival probabilities by level of resting ejection fraction for patients with moderate or severe left ventricular dysfunction treated medically and with CABG .

viable rather than infarcted myocardium. Among patients who receive medical therapy, sudden death occurred more frequently in those patients with PET mismatch compared with those patients without such mismatch (73\% versus $29 \%$ ). In these patients, sudden 
With PET Mismatch

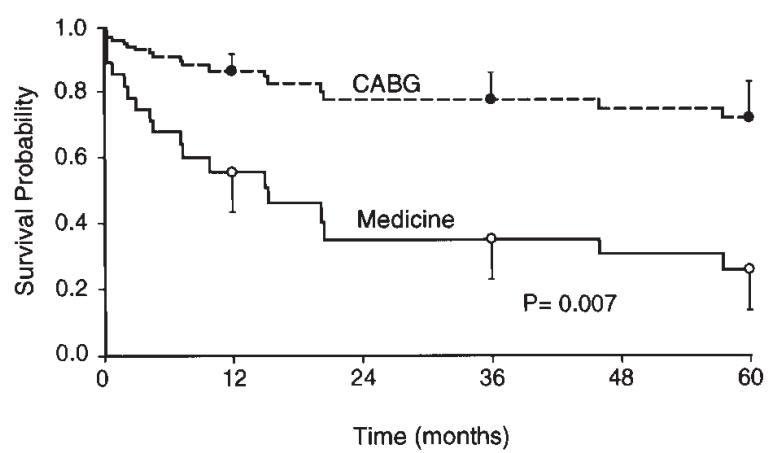

Without PET Mismatch

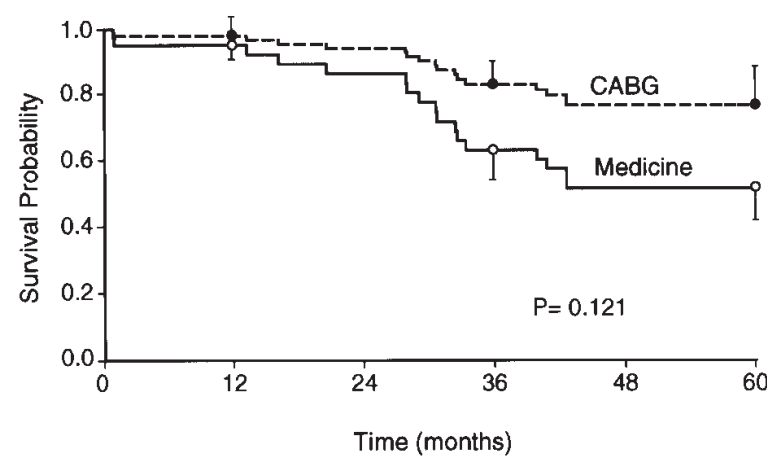

Patients at risk
CABG $\quad 17$

Medicine 33
16
14

25
13

$17-13$

Fig 3. Plot shows adjusted Kaplan-Meier estimated survival probabilities for patients with left ventricular dysfunction treated medically and with CABG by presence or absence of PET mismatch, reflecting hibernating myocardium.

death may be caused by a further reduction in blood supply and/or increased oxygen demand in hibernating regions that ultimately leads to reinfarction and/or lethal arrhythmia.

In contrast, the long-term adjusted survival of patients undergoing revascularization was better than that observed with medical therapy. This finding is consistent with reports of previous cohort and large registry studies. $^{2-4}$ Long-term survival with revascularization was similar in patients with and without evidence of PET mismatch. However, the survival benefit from revascularization over medical therapy was statistically significant only among patients with PET mismatch. In this subgroup of patients, the benefit of revascularization persisted for 5 years of follow-up and actually increased in magnitude throughout this period, corroborating initial observations from our group and others (Fig 3). ${ }^{16-19}$ The operative mortality rate in our study was higher than that reported in some series of patients with low ejection fraction $(<35 \%){ }^{2,3}$ In these reports, the proportion of patients with class III or IV heart failure was also low $(<5 \%)$. In our study, however, $74 \%$ of the surgical patients had low ejection fraction and severe heart failure (Table I). Other reports in patients with low ejection fraction and class III or IV heart failure have found that operative mortality rates range from $5.9 \%$ to $14.9 \%,{ }^{23}$ consistent with that observed in this study. Finally, patients in this study underwent CABG at many centers throughout Southern California with (likely) different levels of expertise in the management of high-risk revascularization. Therefore the operative mortality in our study probably reflects the mortality rate in unselected rather than in highly experienced centers.

Importantly, the survival benefit from revascularization in patients with PET mismatch appeared to be independent of symptoms and was actually greatest among those patients with minimal or no angina. In these patients, CABG improved both anginal and heart failure symptoms. In contrast, in patients without a PET mismatch, CABG appears to improve survival and symptoms only among those patients with limiting angina (Fig 4). Patients with no evidence of viability by PET and minimal or no angina had a lower and similar long-term outcome with revascularization or medical therapy. These findings provide new and important information concerning the value of viability assessment for predicting outcome in patients with severe left ventricular dysfunction, in particular among those patients who have minimal or no angina. Indeed, previous studies found that patients with heart failure and no ischemic symptoms had the same outcome with surgical revascularization as with medical therapy and concluded that these patients should not undergo an operation. ${ }^{2}$ In these studies, however, functional tests of ischemia were not available. Symptoms of cardiac dysfunction, such as dyspnea and heart failure or their hemodynamic correlates, may be the primary clinical manifestation of ischemic myocardium in patients with severely depressed left ventricular function. Our results suggest that patients with 


\section{Without PET Mismatch}

With Severe Angina

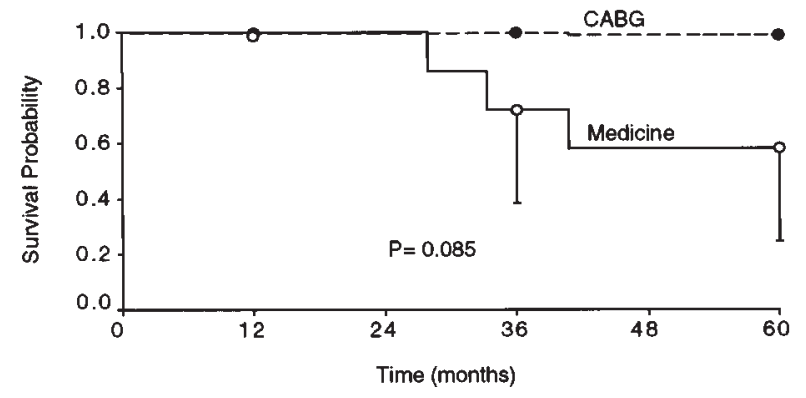

Patients at risk

CABG 7

Medicine 9

6
7

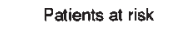

CABG 10

Medicine 24

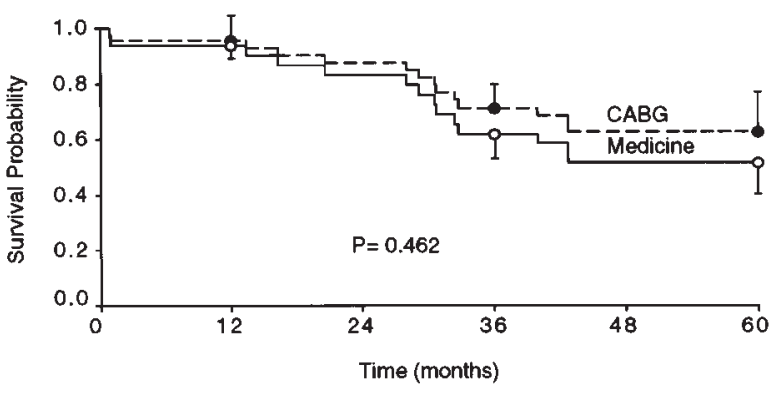

With Mild or no Angina

With PET Mismatch
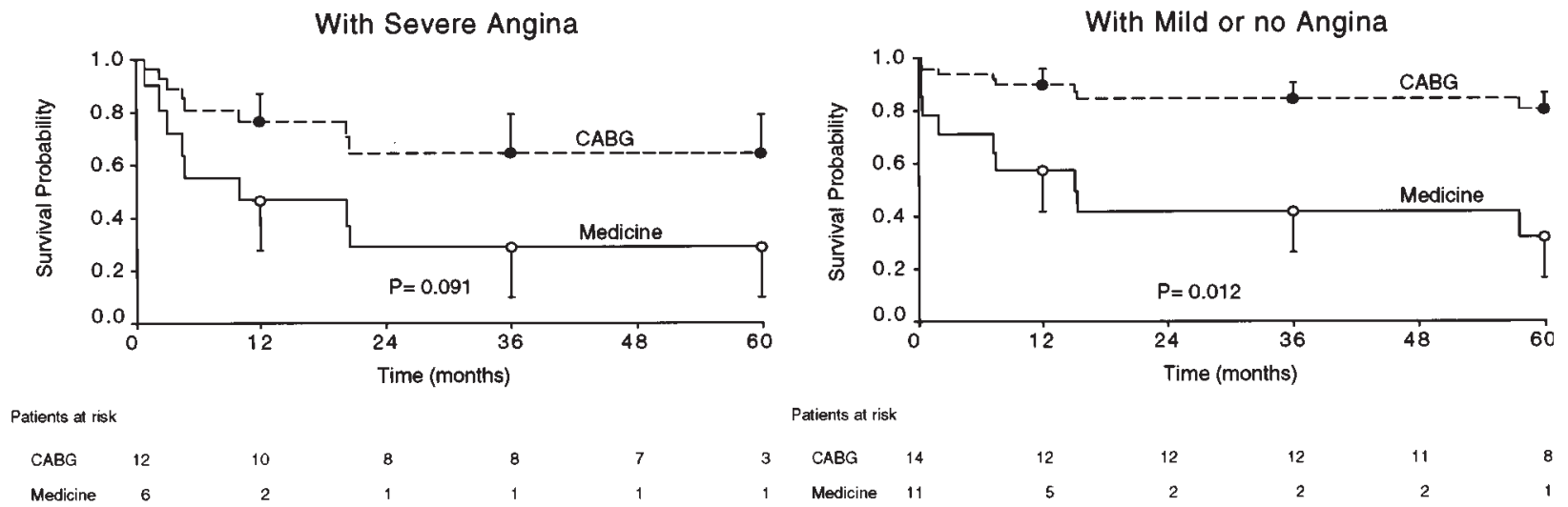

\begin{tabular}{lll}
\multicolumn{3}{c}{ Patients at risk } \\
& & \\
3 CABG & 14 \\
& Medicine & 11
\end{tabular}

12

Fig 4. Plot shows adjusted Kaplan-Meier estimated survival probabilities for patients with left ventricular dysfunction treated medically and with CABG by presence or absence of PET mismatch and by severity of anginal symptoms.

ischemic cardiomyopathy who have minimal or no angina can benefit from revascularization if relatively large areas of viable myocardium can be identified before the operation. In these patients, long-term survival with $\mathrm{CABG}$ appears to be comparable to that achieved with heart transplantation. ${ }^{24}$

Our findings are also important in that they define the subgroup of patients with ischemic cardiomyopathy in whom viability assessment may be critically important for management decisions (Fig 4). Our findings suggest that viability assessment was relatively less important in patients with poor ejection fraction and severe angina, because they appeared to benefit from revascularization regardless of viability information, presumably by improving ventricular function and/or preventing further loss of functioning myocytes. This is in keeping with the extensive surgical literature. ${ }^{5}$ In contrast, our findings suggest that viability assessment was most beneficial among patients with poor ejection fraction and minimal or no anginal symptoms at presentation. Indeed, long-term outcome with revascularization was better only among patients with evidence of viable myocardium by PET, whereas no difference in survival was observed among those without viable myocardium.

\section{Limitations of the study}

This study was not a randomized controlled trial, and the possibility of treatment selection bias exists, even after controlling for important prognostic variables. However, in the absence of randomized studies that compare the outcomes of medical therapy and CABG in patients with low ejection fraction on the basis of the presence or absence of viable myocardium, observational studies such as this are the main source of insight 
concerning these patients. Moreover, it is important to note that multiple observational studies, which use different methods for evaluating myocardial viability, all suggest that short-term outcome in patients with depressed left ventricular function but evidence of viability is better with revascularization than with medical therapy alone. ${ }^{16-20}$ The concurrence of these studies provides more support for this conclusion than the results of any 1 study.

\section{Conclusions}

The present study shows that patients with severe left ventricular dysfunction and evidence of relatively large areas of viable myocardium, as assessed by PET, have improved long-term survival with revascularization as compared with medical therapy. This survival benefit is associated with a significant improvement in angina and heart failure symptoms. Long-term surgical survival benefit and symptomatic improvement in patients without evidence of viability is apparent only among those those with severe angina. These results suggest that viability assessment is important for selecting patients with low ejection fraction who will benefit most from revascularization, in particular if minimal or no anginal symptoms are present.

We thank Ron Sumida, Lawrence Pang, Francine Aguilar, Gloria Stocks, Mark Hulgan, and Der Jenn for their technical assistance in performing the PET studies and Sheila Jackman for her secretarial assistance.

\section{REFERENCES}

1. Emond M, Mock MB, Davis KB, et al. Long-term survival of medically treated patients in the Coronary Artery Surgery Study (CASS). Circulation 1994;90:2645-57.

2. Alderman EL, Fisher LD, Litwin P, et al. Results of coronary artery surgery in patients with poor left ventricular function (CASS). Circulation 1983;68:785-95.

3. Pigott JD, Kouchoukos NT, Oberman A, Cutter GR. Late results of surgical and medical therapy for patients with coronary artery disease and depressed left ventricular function. J Am Coll Cardiol 1985;5:1036-45.

4. Bounous EP, Mark DB, Pollock BG, et al. Surgical survival benefits for coronary disease patients with left ventricular dysfunction. Circulation 1988;78(suppl):I151-7.

5. Baker DW, Jones R, Hodges J, Massie BM, Konstam MA, Rose EA. Management of heart failure. III. The role of revascularization in the treatment of patients with moderate or severe left ventricular systolic dysfunction. JAMA 1994;272:1528-34.

6. Tillisch J, Brunken R, Marshall RC, et al. Reversibility of cardiac wall motion abnormalities predicted by positron tomography. $\mathrm{N}$ Engl J Med 1986;314:884-8.

7. Ragosta M, Beller GA, Watson DD, Kaul S, Gimple W. Quantitative planar rest-redistribution Tl-201 imaging in detection of myocardial viability and prediction of improvement in left ventricular function after coronary artery bypass surgery in patients with severely depressed left ventricular function. Circulation 1993;86:1630-41.

8. Gropler RJ, Geltman EM, Sampathkumaran K, et al. Comparison of carbon-11-acetate with fluorine-18-fluorodeoxyglucose for delineating viable myocardium by positron emission tomography. J Am Coll Cardiol 1993;22:1587-97.

9. Marwick TH, MacIntyre WJ, Lafont A, Nemec JJ, Salcedo EE. Metabolic responses of hibernating and infarcted myocardium to revascularization: a follow-up study of regional perfusion, function, and metabolism. Circulation 1992;85:1347-53.

10. Iskandrian AS, Hakki AH, Kane SA, et al. Rest and redistribution thallium-201 scintigraphy to predict improvement in left ventricular function after coronary artery bypass grafting. Am J Cardiol 1983;51:1312-6.

11. Dilsizian V, Rocco TP, Freedman NM, Leon MB, Bonow RO. Enhanced detection of ischemic but viable myocardium by the reinjection of thallium after stress-redistribution imaging. $\mathrm{N}$ Engl J Med 1990;323:141-6.

12. Rahimtoola SH. The hibernating myocardium. Am Heart J 1989;117:211-21.

13. Braunwald EB, Kloner RA. The stunned myocardium: prolonged, postischemic ventricular dysfunction. Circulation 1982; 66:1146-9.

14. Bolli R. Mechanism of myocardial stunning. Circulation 1990;82:723-38

15. Di Carli M, Asgarzadie F, Schelbert HR, et al. Quantitative relation between myocardial viability and improvement in heart failure symptoms after revascularization in patients with ischemic cardiomyopathy. Circulation 1995;92:3436-44.

16. Di Carli MF, Davidson M, Little R, et al. Value of metabolic imaging with positron emission tomography for evaluating prognosis in patients with coronary artery disease and left ventricular dysfunction. Am J Cardiol 1994;73:527-33.

17. Eitzman D, Al-Aouar Z, Kanter HL, et al. Clinical outcome of patients with advanced coronary artery disease after viability studies with positron emission tomography. J Am Coll Cardiol 1992;20:559-65

18. Lee KS, Marwick TH, Cook SA, et al. Prognosis of patients with left ventricular dysfunction, with and without viable myocardium after myocardial infarction: relative efficacy of medical therapy and revascularization. Circulation 1994;90:2687-94.

19. Gioia G, Powers J, Heo J, Iskandrian AS. Prognostic value of rest-redistribution tomographic thallium-201 imaging in ischemic cardiomyopathy. Am J Cardiol 1995;75:759-62.

20. Williams MJ, Odabashian J, Lauer MS, Thomas JD, Marwick TH. Prognostic value of dobutamine echocardiography in patients with left ventricular dysfunction. J Am Coll Cardiol 1996;27:132-9.

21. Pagley PR, Beller GA, Watson DD, Gimple LW, Ragosta M. Improved outcome after coronary bypass surgery in patients with ischemic cardiomyopathy and residual myocardial viability. Circulation 1997;96:793-800.

22. SPSS advanced statistics. Chicago: Marija J Norusis/SPSS Inc; 1994. TM 6.1.

23. Wechsler AS, Junod FL. Coronary bypass grafting in patients with chronic congestive heart failure. Circulation 1989;79(suppl):I92-6

24. Kobashigawa JA, Laks H, Drinkwater DC, et al. The University of California at Los Angeles experience in heart transplantation. Clin Transpl 1995:129-35. 